\title{
Comparison of Dislocation Mapping Using Electron Channeling Contrast Imaging and Cross-Correlation Electron Backscattered Diffraction
}

\author{
Bret E. Dunlap ${ }^{1}$, Timothy J. Ruggles ${ }^{2}$, David T. Fullwood ${ }^{3}$, Martin A. Crimp ${ }^{1}$ \\ 1. Michigan State University, Chemical Engineering and Materials Science, East Lansing, MI, USA \\ 2. National Institute of Aerospace, Hampton, VA, USA \\ 3. Brigham Young University, Mechanical Engineering, Provo, UT, USA
}

The characterization and quantification of dislocations is predominately carried out using transmission electron microscopy (TEM). Nevertheless, the sample preparation necessary for TEM foils can be cumbersome and possibly introduce defects. Two other techniques for characterizing and quantifying dislocations are electron channeling contrast imaging (ECCI) and cross-correlation electron backscattered diffraction (CC-EBSD). ECCI is similar to TEM in that specific imaging (channeling or diffraction) conditions need to be established, but is carried out in a scanning electron microscope on bulk samples, resulting in less complex sample preparation. CC-EBSD measures subtle changes in orientation measured from EBSD patterns to calculate elastic strains and the Nye tensor to deduce and map the total geometrically necessary dislocation (GND) content. This work presented here compares the capabilities of ECCI and CC-EBSD to map dislocations generated from nanoindentation.

A sample of commercially pure polycrystalline body-centered-cubic Ta was metallographically prepared through a final polishing step of 4 to 1 mixture of Struers OP-S and aqueous $\mathrm{H}_{2} \mathrm{O}_{2}$. Indents were made on the polished surface using a conical-spherical tip with a radius of $\sim 1 \mu \mathrm{m}$ and a maximum load of 4 $\mathrm{mN}$. ECCI and CC-EBSD analysis were carried out on the same indents. The cross-correlation calculations were carried out using the OpenXY software package [1].

Figure 1a presents multiple ECC images stitched together that shows the dislocation content around an indent. Most dislocations appear as dots that have bright/dark contrast, which is typical for dislocations with line directions roughly perpendicular to the bulk sample surface. Figure $1 \mathrm{~b}$ presents a CC-EBSD calculated GND map of the same indent that shows GND content that is qualitatively the same as that shown in the ECC image. In Figure 2a, a higher magnification ECC image, shows dislocations at the top left of an indent, while figure $2 \mathrm{~b}$ shows the corresponding CC-EBSD GND map. Again, qualitatively the dislocation distributions revealed by the two techniques appear the same. Nevertheless, close inspection reveals some discrepancies between the results of the two approaches. In particular, the CC-EBSD appears to miss a number of individual dislocations, but consistently maps areas where ECCI reveals more closely spaced dislocations, suggesting ECCI is better suited for lower dislocation densities and CC-EBSD for higher dislocation densities.

There are a number of potential reasons for the discrepancies between the two approaches. First, the CC-EBSD step size may influence the result; as the step size gets smaller, the GND noise gets higher [2]. CC-EBSD step size may also affect the ability to resolve closely spaced dipole dislocations (statistically stored dislocations-SSDs vs. GNDs), while ECCI can resolve these. Having said that, any given ECC image taken at a particular imaging condition $\mathbf{g}$ may not reveal all of the dislocations in a given region due to some of the dislocations being out of contrast (i.e. to $\mathbf{g} \bullet \mathbf{b}=0$ invisibility). Therefore, ECC imaging must be carried out with multiple channeling conditions to realize the total dislocation content. CC-EBSD is not limited in this manner. 


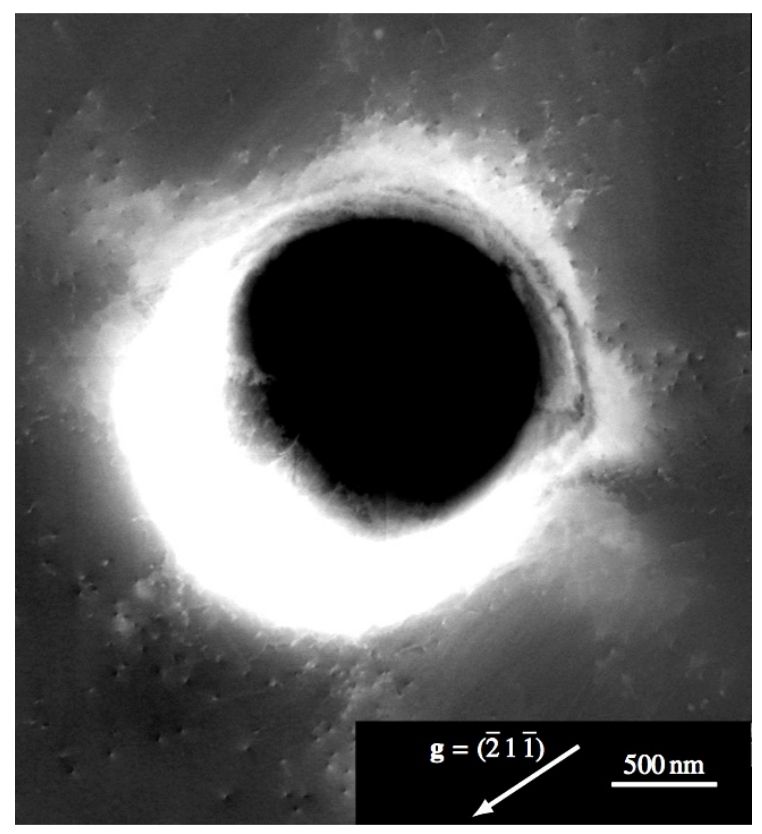

(a)

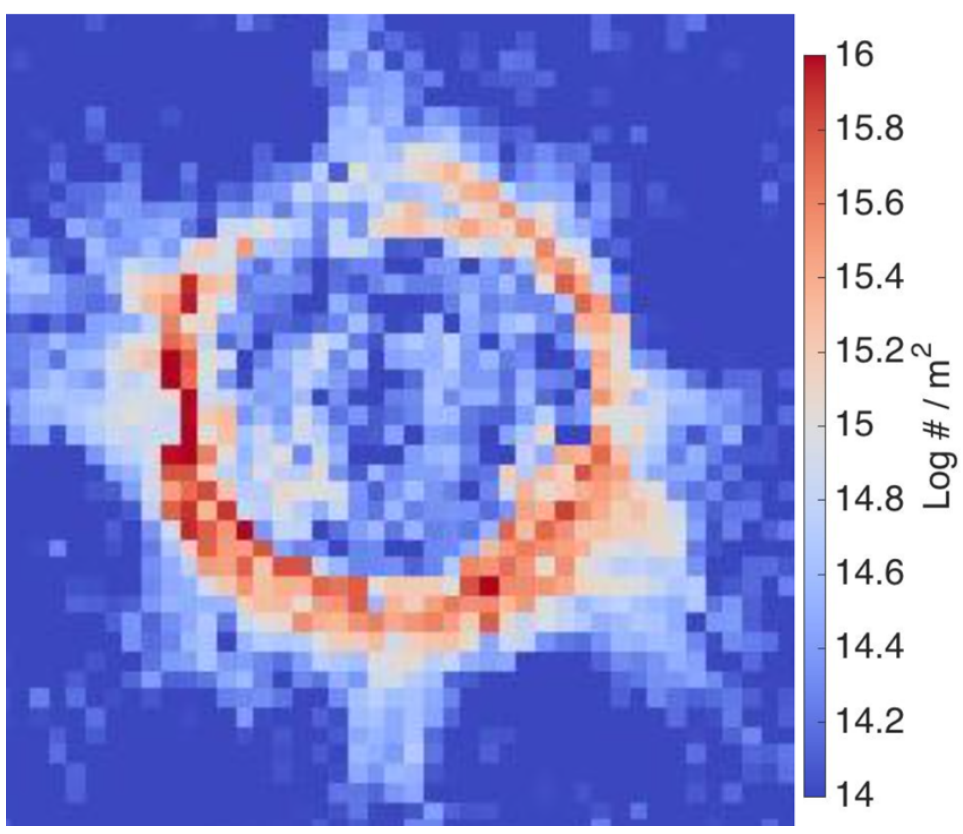

(b)

Figure 1. (a) Multiple ECC images stitched together showing the dislocation distribution around an indent. (b) CC-EBSD calculated GND map showing the dislocation distribution around the same indent.

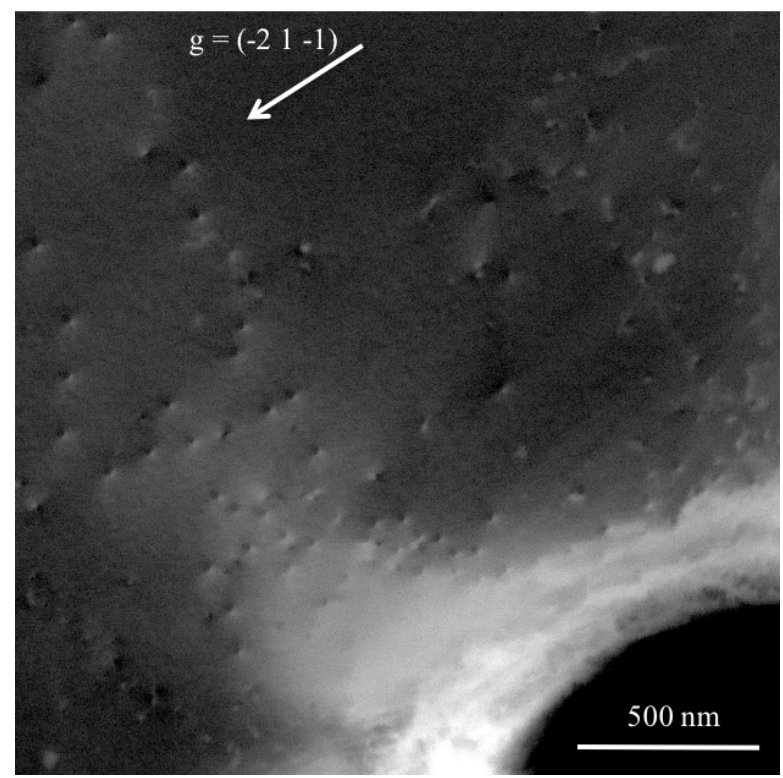

(a)

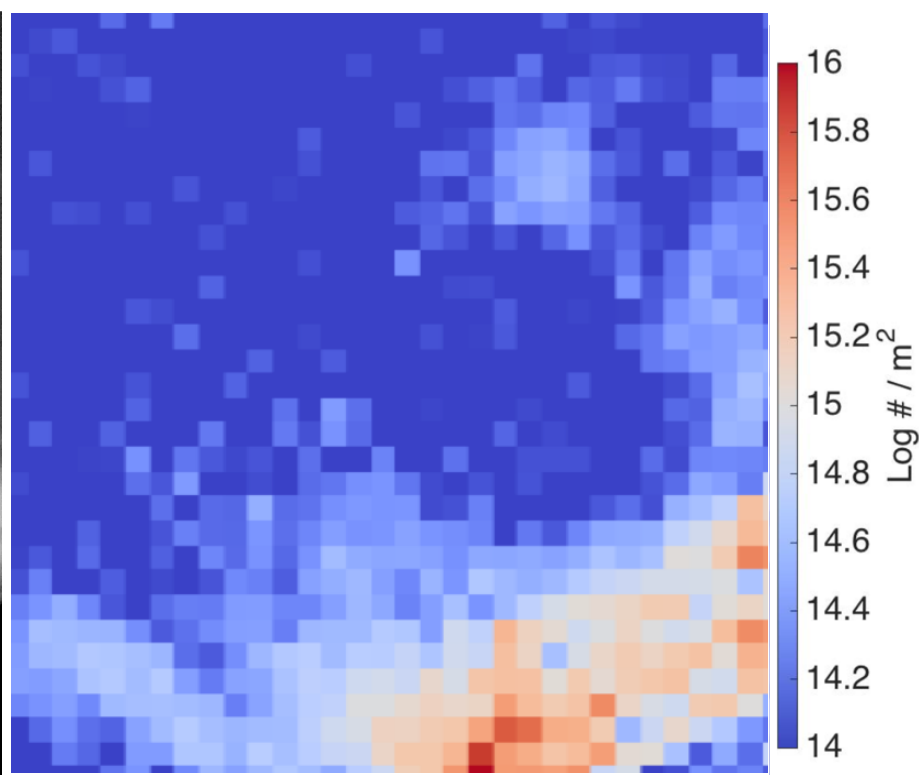

(b)

Figure 2. (a) ECC image showing the dislocation distribution from the upper-left of an indent. (b) CCEBSD calculated GND map showing the dislocation distribution of the same area on the same indent.

\section{References:}

[1] OpenXY, URL: https://github.com/BYU-MicrostructureOfMaterials/OpenXY

[2] B. Adams and J. Kacher, Computers, Materials and Continua 14 (2009), p. 188. 